\title{
Regulation of early growth response-1 (Egr-1) gene expression by Stat1-independent type I interferon signaling and respiratory viruses
}

https://doi.org/10.1515/cmb-2020-0129

Received July 21, 2021; accepted December 13, 2021

\begin{abstract}
Respiratory virus infection is one of the leading causes of death in the world. Activation of the Jak-Stat pathway by Interferon-alpha/beta (IFN- $\alpha / \beta)$ in lung epithelial cells is critical for innate immunity to respiratory viruses. Transcriptional factor profiling in the transcriptome and RNA analysis revealed that Early growth response-1 (EGR1/Egr-1) was rapidly induced by IFN- $\alpha / \beta$ and Toll-like receptor (TLR) ligands in multiple cell types. Studies in mutant cell lines lacking components of the interferon-stimulated gene factor complex (ISGF-3) revealed that IFN- $\beta$ induction of Egr-1 was independent of Stat1, Stat2, or Irf9. Activation of the Mek/Erk-1/2 pathway was implicated in the rapid induction of Egr-1 by IFN- $\beta$ in serum-starved mouse lung epithelial cells. Interrogation of multiple microarray datasets revealed that respiratory viruses including coronaviruses induced IFN- $\beta$ and regulated Egr-1 expression in human lung cell lines. Furthermore, bioinformatic analysis revealed that type I interferon-stimulated genes and Egr-1 inducible genes including transcription factors, mediators of cell growth, and chemokines were differentially regulated in the human lung cell lines after coronavirus infection, and in the lung biopsies of COVID-19 patients.
\end{abstract}

Keywords: Interferon- $\alpha / \beta$, Innate immunity, Early Growth Response-1. coronaviruses, COVID-19

MSC: $00,68,92$

\section{Introduction}

Interferons (IFNs) are pleiotropic cytokines that play a central role in innate and adaptive immunity $[1,2]$. There are 3 major types of interferons. The type I interferons consists of IFN-alpha/beta (IFN- $\alpha / \beta$ ), type II interferon represented by interferon-gamma (IFN- $\gamma$ ), and type III interferons represented by interferon lambda (IFN- $\lambda$ ). The biological effects of IFN are mediated mainly by the rapid and dramatic changes in gene expression [3]). Type I IFN signaling involves the binding of IFN- $\alpha / \beta$ to its receptor (IFNAR) and activation of receptor-associated Janus protein tyrosine kinases Jak1 and Tyk2 and the phosphorylation of Stat1 and Stat2 to form a heterodimer. This heterodimer associate with the Interferon responsive factor 9 (Irf9) to form the interferon-stimulated gene factor-3 (ISGF-3) complex on interferon-stimulated response elements (ISRE) in the promoters of type I IFN responsive genes to regulate transcription $[1,2,4]$. Stat 1 homodimer or heterodimer of Stat1/Stat2 can also bind to Gamma activated sequence (GAS) in the promoter and regulate transcription of

\footnotetext{
*Corresponding Author: Chilakamarti V. Ramana: Department of Medicine, Dartmouth-Hitchcock Medical Center, Lebanon, NH 03766, USA

Department of Stem Cell and Infectious Diseases, KaviKrishna Laboratory, Guwahati Biotech Park, Indian Institute of Technology, Guwahati, India

Thoreau Laboratory for Global Health, University of Massachusetts, Lowell, MA 01854, USA, E-mail: ramana181@gmail.com Bikul Das: Department of Stem Cell and Infectious Diseases, KaviKrishna Laboratory, Guwahati Biotech Park, Indian Institute of Technology, Guwahati, India

Thoreau Laboratory for Global Health, University of Massachusetts, Lowell, MA 01854, USA
} 
some type I IFN responsive genes $[1,4]$. In addition to this classical canonical Jak-Stat pathway, non-canonical pathways involving Stat2, Irf9, and unphosphorylated ISGF-3 components in the regulation of gene expression have been described [5]. Furthermore, several signal transduction pathways are activated by IFN receptors in parallel to Jak-Stat including extracellular signal-regulated kinases (Erk-1/2) and phosphoinositide 3'-kinase/Akt pathways [6,7]. The first wave of Interferon signaling is followed by induction of transcription factors such as Interferon regulatory factors (IRFs) that sustain the secondary and tertiary transcriptional responses. TLR recognition of pathogens by immune cells results in the production of multiple cytokines such as IFN- $\alpha / \beta$, Tumor Necrosis Factor- $\alpha$ (TNF- $\alpha$ ), and Interleukin- $\beta$ (IL-1 $\beta$ ) in innate immunity [8]. Activation of multiple signal transduction pathways and cross-talk between the pathways enables fine-tuning of gene expression in innate immunity [9]. Cross-talk between TNF- $\alpha$ and IFN signaling pathways regulate inflammatory gene expression to influence the immune responses [10,11]. Clinical significance of the balance between immune modulation and inflammation in signal transduction pathways has been demonstrated in a variety of autoimmune diseases [12, 13].

Early growth response 1 (EGR1/ Egr-1) belongs to a family of immediate-early response genes that contain a conserved zinc finger DNA-binding domain and binds to a GC-rich sequence in the promoters of target genes [14]. A variety of signals, including serum, growth factors, cytokines, and hormones stimulate Egr-1 expression $[15,16]$. Egr-1 has been shown to play an important role by regulating inflammatory gene expression in a variety of lung diseases and in mouse lung injury models including asthma, emphysema, airway inflammation, and pulmonary fibrosis $[17,18,19]$. Ischemia-mediated activation of Egr-1 triggers the expression of pivotal regulators of inflammation including the chemokine, adhesion receptor, and pro-coagulant gene expression [20]. Egr-1 stimulates chemokine production in interleukin-13 mediated airway inflammation, and remodeling in the lung [21]. High levels of expression of Egr-1 and Egr-1 inducible genes were reported in atherosclerosis, an inflammatory disease [22]. Egr-1 may have a potential role in liver injury and in acute pancreatitis [23, 24, 25]. Lipopolysaccharide (LPS) induction of Egr-1 was mediated by the activation of Erk-1/2 pathway and serum response elements [26]. In this study, transcription factor profiling in interferon-mediated gene expression data sets and RT-PCR revealed that Egr-1 was rapidly induced by IFN- $\alpha / \beta$ and TLR ligands in multiple cell types. Studies in mouse and human fibroblast mutant cell lines revealed that Egr-1 induction by type I interferons was independent of transcription factors Stat1, Stat2 or Irf9. Furthermore, the regulation of Egr- 1 by IFN- $\beta$ was mediated by the activation of the Erk-1/2 pathway in serum-starved mouse lung epithelial cells. Respiratory pathogens including coronaviruses (SARS-CoV-1 and 2) and influenza viruses regulated the expression of Egr-1 in human lung cell lines and in lung biopsies of COVID-19 patients. These studies suggest that the regulation of Egr-1 may play an important role in the antiviral response and inflammatory disease.

\section{Materials and Methods}

\section{Gene expression datasets and Bioinformatics}

Gene expression in response to Interferon, TNF- $\alpha$, and TLR agonist treatment in human peripheral blood mononuclear cells (PBMC), human hepatoma cells (Huh-7), mouse bone marrow-derived macrophages (BMDM) were reported previously [27, 28, 29]. The GEO dataset accession numbers for PBMC and Huh-7 were GSE17762, GSE48400 respectively. Supplementary data was downloaded from the Journal publisher websites. GEO datasets were analyzed with the GeoR2R method (NCBI, GEO datasets, National Institute of Health, USA). Gene expression datasets representing human lung cell lines infected with respiratory viruses and from COVID-19 patients were reported previously [30, 31]. The GEO dataset accession numbers for human lung epithelial cells infected with SARS-CoV2 and SARS-CoV-1 were GSE147507, GSE 148729, and GSE47961. Three samples for each condition were used. Cluster analysis of gene expression data by linkage across arrays and genes was performed using Heatmapper gene expression software tools (www.heatmapper.ca). Outliers of expression were not included in the analysis. Virus infection or interferon treatment induces a signature of interferon-regulated gene expression (internal controls). The datasets that do not fulfill internal controls that the experiment was successful were removed from the analysis. Time-course data were examined to deter- 
mine that EGR1 was maximally induced in lung epithelia cells by SARS-CoV1 and SARS-CoV-2 at 24 hours post-infection (Supplementary Figure 1 and 2). Gene expression resources from Immgen RNA seq SKYLINE (http://rstats.immgen.org/Skyline_COVID-19/skyline.html) and Metascape ( https://metascape.org/COVID/) were used. Protein interactions were interrogated and visualized in the Search Tool for the Retrieval of Interacting Genes and proteins (STRING) and BIOGRID databases. Biogrid software program e-syN was used to generate protein interaction network. Transcription factor and interferon data was retrieved from TRUUST (https://www.grnpedia.org/trrust/) and Interferome (www.interferome.org), respectively.

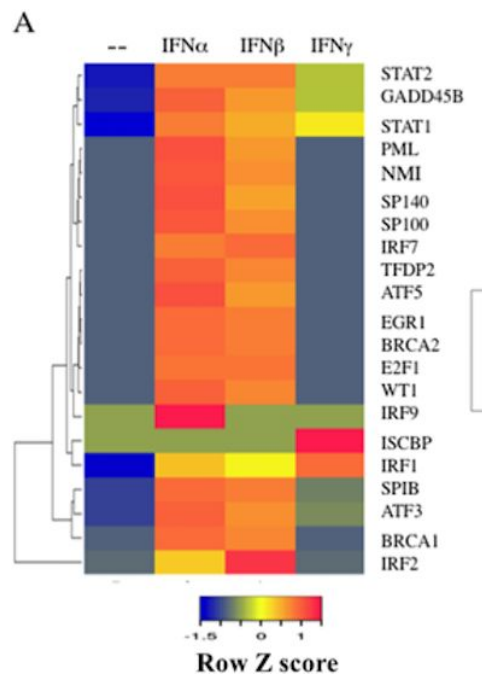

B

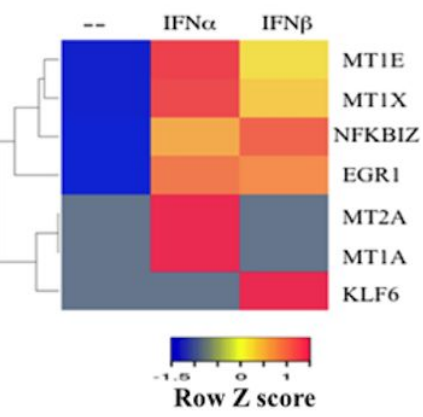

Figure 1: Regulation of transcription factor (TF) mRNA levels in human peripheral blood mononuclear cells (PBMC) and human hepatoma (Huh7) cells by interferon treatment (A) Transcription factor expression profiles were retrieved from microarray datasets of human PBMC treated with IFN- $\alpha$, IFN- $\beta$ or IFN- $\gamma$ for $0.5-2$ hours (B) Transcription factor expression in Huh7 cells treated with IFN- $\alpha$ or IFN- $\beta$ for 0.5 hours were retrieved from the microarray datasets using the GEO R2R analysis. Cluster analysis of gene expression was performed using the heatmapper software.

A

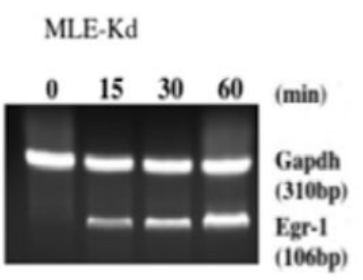

C

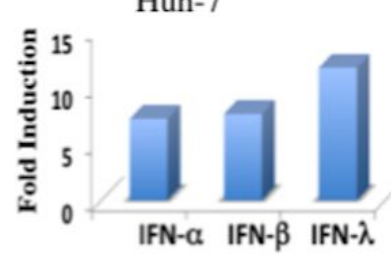

B

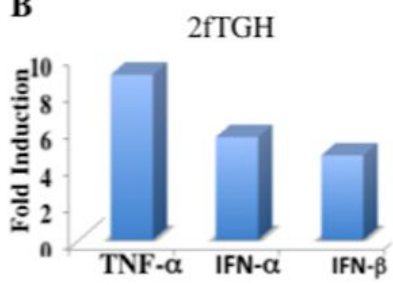

Figure 2: Regulation of Egr-1 mRNA levels by Interferon-beta (IFN- $\beta$ ) in mouse and human cells. (A) Mouse MLE-Kd cells were treated with IFN- $\beta(1000 \mathrm{U} / \mathrm{ml})$ and RNA was prepared from cells at $0,0.25,0.5$, and 1 hour after treatment. RNA levels of Egr-1 and GAPDH were determined by RT-PCR analysis. (B) Human 2 fTGH were treated with TNF- $\alpha(20 \mathrm{ng} / \mathrm{ml})$, IFN- $\alpha(1000 \mathrm{U} / \mathrm{ml})$ or IFN$\beta(1000 \mathrm{U} / \mathrm{ml})$ for 2 hours. Egr-1 and $\beta$-Actin levels were determined by RT-PCR in control and treatment and expressed as fold induction. (C) Regulation of Egr-1 in Huh7 cells in response to IFN- $\alpha$, IFN- $\beta$, and IFN- $\lambda$. Fold change values of Egr- 1 were retrieved from GEO datasets using the GEO R2R analysis. 


\section{Cell culture and cytokine treatment}

Mouse lung epithelial (MLE-Kd) and macrophage (RAW264.7) cell lines were used [11, 32]. Human fibrosarcoma cell line (2fTGH) and mutant cell lines lacking Stat1 (U3A), Stat2 (U6A), and IRF9 (U2A) were described previously [33]. Wild -type and Stat1-knockout mouse embryo fibroblast (MEF) cell lines were used [34]. Cells were maintained in DMEM supplemented with $10 \% \mathrm{FBS}$ and $1 \%$ penicillin and streptomycin. Cells were plated at $60-70 \%$ density and maintained in full medium for a day. Cells were incubated in serum-free medium for another 24 hours. Cells were treated with TNF- $\alpha(20 \mathrm{ng} / \mathrm{ml})$ or IFN- $\beta(1000 \mathrm{U} / \mathrm{ml})$ for the indicated time. Cytokines were purchased from PBL Assay Science (Nutley, NJ). RAW 264.7 cells were treated with PolyIC $(10 \mu \mathrm{g} / \mathrm{ml})$, LPS (10ng/ml) or IFN- $\beta$ (1000U/ml). PolyIC and LPS were purchased from InvivoGen (San Diego, CA) and used according to the manufacturer's instructions.

\section{RNA Expression and Western blot Analysis}

RNA was extracted from cell pellets using the Qiagen RNeasy kit (Valencia, CA). Reverse transcriptasepolymerase chain reactions (RT-PCR) were performed using Ambion Retroscript (Austin, TX), according to the manufacturer's protocol. Primer sequences for Egr-1, Irf1, and Gapdh were obtained from the molecular reagents section of Mouse Genome Informatics (MGI). Human Egr-1 and $\beta$-Actin primer sequences were previously described (22). PCR products were resolved on a $1 \%$ agarose gel containing ethidium bromide and visualized with U.V. Light and images were captured on a digital system. Image files were processed with ImageJ (NIH) software. Specific gene expression was normalized to GAPDH or $\beta$-Actin and fold changes in the treated samples were calculated with respect to controls. At least, three samples were used for each condition. MLE-Kd cell extracts were prepared and proteins were separated by electrophoresis using $8 \%-10 \%$ SDSPAGE gels. Proteins in the gel were electrophoretically transferred to polyvinylidene difluoride membranes (Bio-Rad, CA) and subjected to immunoblotting with the antibodies for phosphorylated Erk-1/2 (Thr202/Tyr 204) or total Erk-1/2 from Cell Signaling Technology (Beverly, MA). Blots were visualized by enhanced chemiluminescence western detection system (Pierce, IL). Western blot was repeated three times.

\section{Results and Discussion}

\subsection{Regulation of Egr-1 by Interferons and Toll-Like Receptor (TLR) ligands}

The role of phosphorylation cascade and activation of the Jak-Stat pathway in IFN- $\alpha / \beta$ signaling is well established [1, 2]. In addition, inducible transcription factors such as Irf1, Irf2, Irf7 also play an important role in sustaining and modulating the IFN- $\alpha / \beta$ signaling $[4,5]$. Transcription factor profiling in the transcriptome provides novel insights into the functional organization and connectivity in signal transduction pathways in eukaryotic cells [35]. A large number of microarray studies on interferon-regulated gene expression were limited by factors such as cell type, long delay after treatment (2-24 hours) or treatment in cell culture media with high serum. These studies may have missed earlier dynamic changes in transcription factors and growthregulated genes. In order to identify novel inducible transcription factors regulated by Interferon $\alpha / \beta$, gene expression datasets representing early time points (0.5-2 hours) were examined [27, 29]. A wide variety of transcription factors were induced by IFN- $\alpha / \beta$ in human peripheral blood mononuclear cells (PBMC) and in Huh7 hepatoma cells (Figures 1A and 1B). Several Metallothionein (MT) isoforms were induced in the early transcription response by IFN- $\alpha / \beta$ in Huh7 liver cells. Metals and inflammatory stimuli are the major inducers of Metallothionein in the lung and liver. Early growth response-1 (Egr-1) was rapidly induced by IFN- $\alpha / \beta$ in both cell types and selected for further studies. RNA expression studies in mouse lung epithelial (MLE-Kd) and human fibrosarcoma (2fTGH) cell lines confirmed that IFN- $\alpha / \beta$ induced Egr-1 (Figure 2A and 2B). These results were consistent with previous studies in human fibroblasts (15). TNF- $\alpha$ induction of Egr-1 was much higher than IFN $\alpha / \beta$ in 2fTGH cells (Figure 2B). Interestingly, TNF- $\alpha$ but not IFN- $\alpha$ induced Egr-1 mRNA in Hela cells suggesting differential pathway regulation [15]. Furthermore, IFN- $\lambda$ induction of Egr-1 was higher than with IFN- $\alpha / \beta$ in Huh7 cells (Figure 2C). TLR recognition of viral and bacterial components such as doublestranded RNA (Poly IC) and LPS activate diverse signal transduction pathways, stimulate the production of 
type I interferons and activate a large number of genes including interferon-stimulated gene expression [36]. TLR4 ligand (LPS) and TLR3 ligand (Poly IC) induced Egr-1 mRNA in RAW264.7 mouse macrophage cells. Interestingly, LPS treatment resulted in the rapid and enhanced activation compared with Poly IC or IFN- $\beta$ treatment in RAW 264.7 cells (Figure 3A). Time-course experiments in bone marrow derived-macrophages (BMDM) revealed distinct Egr-1 gene expression profiles for each treatment (Figures 3B-3D). These studies revealed that cytokines and TLR ligands use multiple and distinct signal transduction pathways to induce Egr-1 gene expression. Factors such as diversity, intensity and duration of the signal transduction pathways might account for the variation in Egr-1 expression levels in response to different cytokines and TLR ligands.

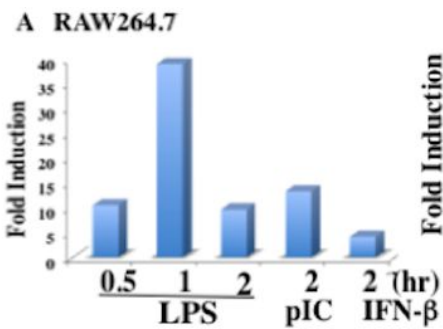

C $\mathbf{B M D M}(\mathbf{L P S})$

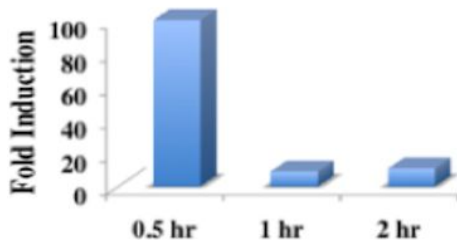

B BMDM (Poly IC)

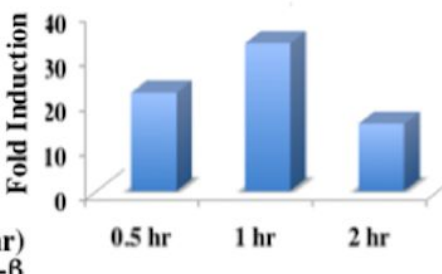

D $\operatorname{BMDM}(\mathrm{IFN}-\beta)$

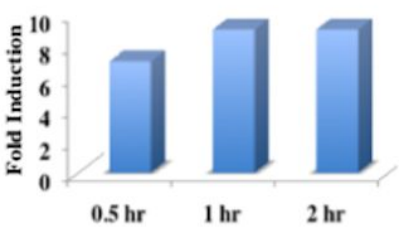

Figure 3: Regulation of Egr-1 mRNA levels in mouse macrophages by TLR ligands and IFN- $\beta$. (A) Mouse macrophage (RAW264.7) cells were treated with LPS for $0.5-2$ hours or with poly IC or IFN- $\beta$ for 2 hours. Egr- 1 and GAPDH levels were determined by RTPCR analysis in control and treatment and expressed as fold induction. (B-D) Egr-1 mRNA levels in BMDM cells treated with poly IC, LPS, or IFN- $\beta$ for 0.5 to 2 hours. Egr-1-fold induction data were obtained from the reference 28 .

A

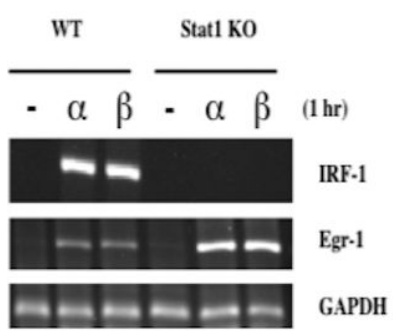

B

Egr-1 induction be IFN- $\beta$ in mouse and human cell lines
\begin{tabular}{|l|l|l|}
\hline Cell line & Missing protein & Egr-1 induction \\
\hline & & by IFN- $\beta$ \\
\hline WT MEF & None & + \\
\hline Stat1 KO MEF & Stat1 & + \\
\hline 2 fTGH & None & + \\
\hline U3A & Stat1 & + \\
\hline U6A & Stat2 & + \\
\hline U2A & IRF9 & + \\
\hline
\end{tabular}

Figure 4: Regulation of Egr-1 mRNA levels by IFN- $\beta$ in mouse and human mutant cell lines. (A) Wild-type and Stat1 KO MEF cells were left untreated or treated with IFN- $\alpha$ or IFN $-\beta(1000 \mathrm{U} / \mathrm{ml})$ ?for one hour. RNA levels of Irf1, Egr-1, and GAPDH were determined by RT-PCR analysis (B) Summary of Egr-1 mRNA regulation by IFN- $\beta$ in wild-type and Stat1 KO MEF cells and in human wild-type (2FTGH), Stat1-null (U3A), Stat2-null (U6A), and IRF9-null (U2A) fibrosarcoma cell lines. 


\subsection{Regulation of Egr-1 by IFN- $\beta$ was independent of Stat1 or ISGF-3 components and mediated by the activation of Erk-1/2 pathway}

IFN- $\alpha / \beta$ activates transcription factor complexes including Stat1 homodimer, Stat1-Stat2 heterodimer, and Stat1-Stat2-Irf9 trimeric complex (ISGF-3) that are involved in gene regulation [1, 2]. However, IFN- $\gamma$ - mediated induction of Egr-1 was Stat1-independent in mouse embryonic fibroblasts [34]. Studies in wild-type and Stat1-KO mouse fibroblasts showed that IFN- $\alpha / \beta$ induction of Egr-1 was independent of Stat1 (Figure 4A). In contrast, Irf1 induction by IFN- $\alpha / \beta$ was strictly Stat1-dependent. Furthermore, Egr-1 induction was 3-fold higher in Stat1-mull cells compared with wild-type cells. This enhanced expression of Egr-1was also observed in Stat1 KO fibroblasts in response to IFN- $\gamma$ [34]. Studies in mutant cells lacking Stat1 (U3A), Stat2 (U6A), and Irf9 (U2A) demonstrated that IFN- $\beta$ induction of Egr-1 was independent of ISGF-3 components (Figure 4B). TNF- $\alpha$ and TLR ligands activate several Mitogen-activated protein kinase (MAPK) pathways such as Extracellular signal-regulated kinase (ERK), Jun N-terminal kinase (JNK) and p38 [37]. Activation of the Erk-1/2 pathway leading to the phosphorylation of transcription factors such as Elk1 and Srf1 was implicated in the rapid induction of Egr-1 in response to multiple stimuli $[26,38]$. Western blot analysis revealed that IFN- $\beta$ rapidly activated Erk-1/2 within 30 minutes in serum-starved MLE-Kd cells (Figure 5A). Furthermore, pretreatment with Mek inhibitor (U0126) inhibited the induction of Egr-1 mRNA (Figure 5B). Pretreatment with an equal volume of DMSO (vehicle) has no significant effect on Egr-1induction. Activation of Jak1 and MAPK-interacting kinase (Mnk1) were required for the activation of Erk-1/2 in IFN- $\beta$ signaling $[39,40]$. There are technical complications in the analysis of Erk-1/2 phosphorylation and activation in cell lines including constitutive activation in transformed cells as well as cell culture conditions such as serum and nutrients [39, 40, 41]. Erk-1/2 activates transcription factors such as Elk1 and Srf1 that bind to Ets and serum response elements (SRE) respectively, and mediate transcriptional activation of Egr-1 in response to serum and LPS [26, 38]. Mouse Egr-1 gene promoter contains multiple SRE elements as well as Activator protein (AP1), Cyclic AMP response (CRE), and Ets elements upstream of the transcription start site (Figure 5C). Activation of Srf1 and Elk1 by phosphorylation and binding of Srf1 and Elk1 to the Egr-1 gene promoter in response to IFN- $\beta$ in MLE-Kd cells remains to be established. Expression of Srf1 enhanced the mRNA levels of several interferon-stimulated genes in response to Interferon- $\alpha / \beta$ in mouse macrophages [42]. Detailed promoter analysis revealed that multiple distal and proximal cis-elements were involved in Egr-1 induction [43].

\subsection{Regulation of genes by Interferon- $\alpha / \beta$ and TNF- $\alpha$ in Bone marrow-derived macrophages (BMDM) and peripheral blood monocytic cells (PBMC)}

The generation of an inflammatory response is a complex process involving multiple cytokines acting in parallel and in concert in innate and adaptive immunity [36, 44]. Influenza virus-infected dendritic cells and macrophages, which reside in close proximity to lung epithelium, can produce significant amounts of TNF- $\alpha$ and type I IFN in response to virus infection [45]. IFN- $\alpha / \beta$ is involved in signaling cross-talk with TNF- $\alpha$ that enhances or dampens the severity of inflammatory response $[9,10]$. Interaction between Interferon $-\alpha / \beta$ and TNF- $\alpha$ signaling was reported in autoimmune diseases [12, 13]. A select list of genes induced by both TNF- $\alpha$ and Interferon- $\alpha / \beta$ in PBMC and BMDM gene expression datasets were identified from PBMC gene expression dataset (Figure 6). TNF- $\alpha$ induction of these genes was much higher than IFN $\alpha / \beta$ in the mouse BMDM cells. Interestingly, Egr-1 was induced by both cytokines in PBMC and BMDM. These genes are involved in transcriptional regulation (Egr-1, Egr-2, Irf1, Nfkbiz, Nfkbia and Bcl3) and integrating of signal transduction pathways (WT1, BRCA2, NR3C1) that are likely to play an important role in the cytokine storm and shift to hyperinflammatory gene expression in response to coronavirus infections [30, 65]. 


\subsection{Regulation of Egr-1 target genes in PBMC and BMDM by IFN-a/ $\beta$}

Egr-1 is a zinc finger transcription factor that binds to a GC-rich sequence in the promoter regions of target genes. Genes regulated by Egr-1 were identified from the databases such as TRUUST transcription factor database, GEO datasets of wild type and Egr-1 KO cells, and published studies in different cell types and under different stimuli [20, 21, 22, 46]. Egr-1 regulates genes involved in cell growth (Egr2, Atf3, Pdgfrb, Cdkna1, Ccnd1, Tnfsf10, and chemokines involved in inflammation such as (Cxcl10, Cxcl2, Ccl2, Ccl3). It is important to note that many of the Egr-1 target genes were also known targets of NF-KB, AP1, and Stat1 in cytokine signaling [46]. Furthermore, Egr-1 interacts with several other transcription factors such as Ets1, Ets2, Elk1 that are common in cytokine responsive genes [46]. Previous studies have shown that transcription factors of innate and adaptive immunity show functional connectivity involving shared interacting protein partners and target genes [47]. Cluster analysis revealed that Egr-1 induction was correlated with the expression of Egr-1 target genes by IFN- $\alpha / \beta$ in PBMC and in BMDM cells (Figure 7).

A

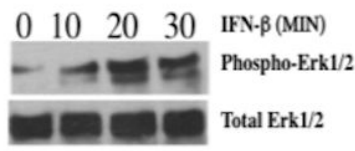

B

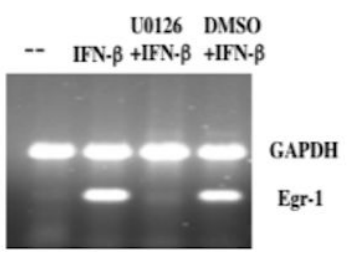

C

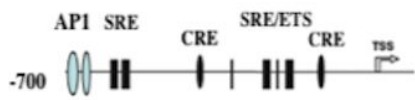

Egr-1 gene promoter

Figure 5: Activation of extracellular signal-regulated kinases (Erk1/2) by IFN- $\beta$ in MLE-Kd cells (A) MLE-Kd cells were treated with IFN- $\beta$ for $0,10,20$ or $30 \mathrm{~min}$. Cell extracts were prepared and the protein levels of phosphorylated Erk-1/2 and total Erk-1/2 were determined by western blot analysis. (B) MLE-Kd cells were left untreated or pre-treated with $40 \mu \mathrm{M}$ of Mek-1 inhibitor U0126 or with an equal volume of DMSO for 2 hours. Cells were stimulated with IFN- $\beta$ alone or after pretreated with DMSO or U0126 for an additional $60 \mathrm{~min}$. Total RNA was isolated and Egr-1 and GAPDH mRNA expression levels were determined by RTPCR analysis. (C) Schematic representation of the mouse Egr-1 gene promoter. Location of the AP1, CRE, SRE, and Ets elements were shown. TSS represents the transcription start site. The map is not to scale.

\subsection{Regulation of Egr-1 by Severe Acute Respiratory Syndrome Coronaviruses (SARS-CoV) and other Respiratory Viruses}

Severe acute respiratory syndrome coronaviruses (SARS-CoV) and influenza viruses are major respiratory pathogens in humans with seasonal epidemics and potential pandemic threats [48, 49]. Respiratory virus infection results in lung injury, a significant component of which was mediated by the host immune response [50]. The highly pathogenic 1918 Influenza virus-induced gene expression signature in mouse lungs characterized by enhanced expression of cytokines such as TNF- $\alpha$, IFN- $\gamma$, and IL- 6 resulting in a dramatic increase in chemokines and inflammatory cell influx consisting of neutrophils, macrophages, and lymphocytes [51, 52]. Gene expression profiling in the lungs of mice infected with 1918 influenza HINI showed enhanced expression of Egr-1 and its downstream target genes Cxcl2 and Ccl2 involved in neutrophil and macrophage inflammatory influx, respectively [51]. Activation of multiple signal transduction pathways leading to the activation of transcription factors such as AP1 and NF-kB in response to influenza infection were 

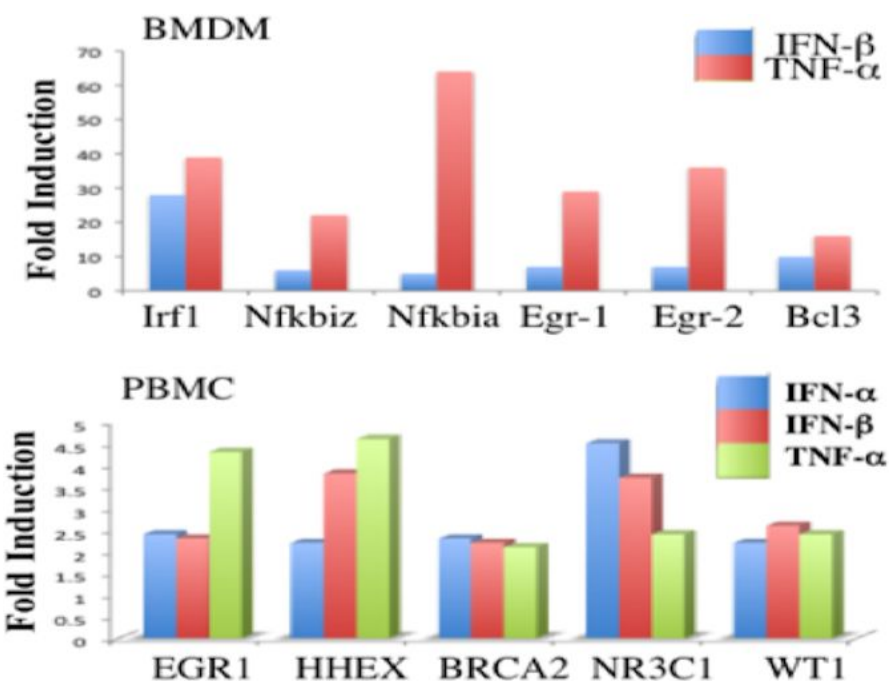

Figure 6: Identification of genes involved in transcriptional regulation and signaling pathways regulated by Interferon- $\alpha / \beta$ and TNF- $\alpha$ in human PBMC and mouse BMDM cells. Gene expression profiles in PBMC (0.5-2 hours) and BMDM (0.5-2 hours) were retrieved from published array datasets.
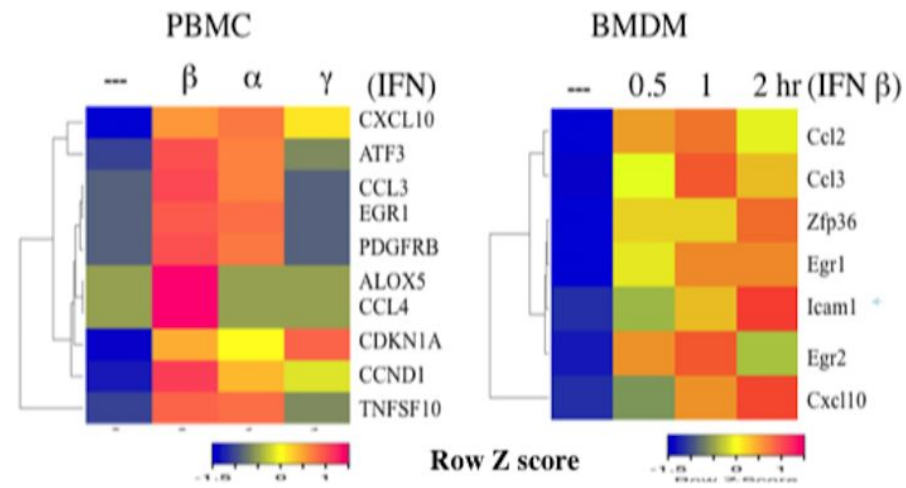

Figure 7: Regulation of mRNA levels of Egr-1 target genes by interferon treatment in human PBMC and mouse BMDM cells (A) Egr-1 target gene expression in human PBMC treated with IFN- $\alpha$, IFN- $\beta$ or IFN- $\gamma$ for 2 hours (B) Egr-1 target gene expression in mouse BMDM cells treated with IFN- $\beta$ for 0.5-2 hours. Gene expression profiles were retrieved from published array datasets. Cluster analysis was performed using the heatmapper software.

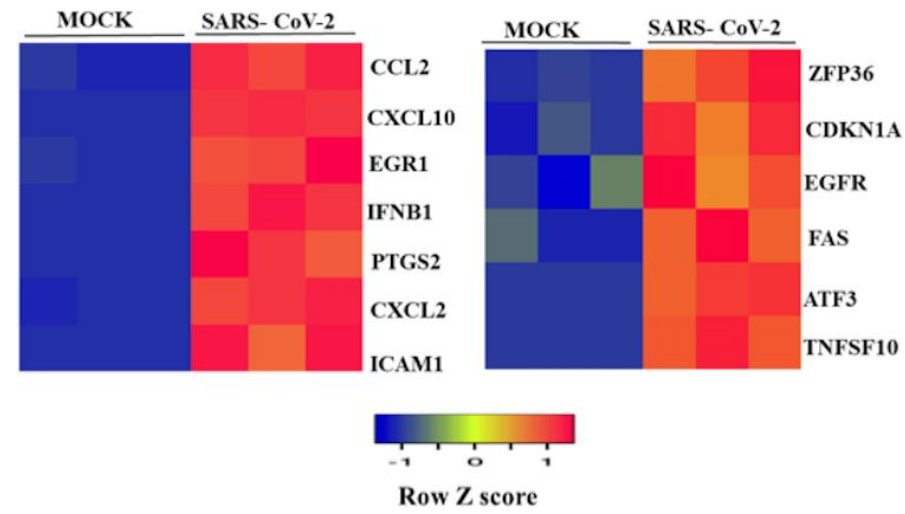

Figure 8: Regulation of IFN- $\beta$ and the interferon-stimulated genes (ISG) mRNA by Coronaviruses in Calu- 3 cells. Calu- 3 cells were mock- infected or infected with the virus SARS-CoV-2 for 24 hours. RNA expression levels were normalized by DEseq2. Data from three samples for each condition were shown. 
reported [53,54]. Influenza virus propagation was impaired by inhibition of the Mek/Erk pathway and lungspecific expression of active Raf kinase results in increased mortality of influenza virus- infected mice [55, 56]. Enhanced cytokine and chemokine expression were also reported in longitudinal studies of severe COVID -19 patients [57, 58]. Gene expression profiling studies of human lung epithelial cell lines such as Calu-3, A549, and NHBE1 in response to respiratory viruses were reported recently [30, 31]. Interrogation of Microarray data sets revealed that Egr-1 was induced by SARS CoV-2 infection but not by mock-infection in Calu-3 cells. This induction was observed in Calu-3 cells at 24 hours after infection in two independent GEO datasets (Figure 8 and Suppl. Figure 1). Egr-1 regulated genes such as chemokines (CXCL10, CXCL2, and CCL2), cell growth (ATF3, CDKN1A, and TNFSF10) and inflammation (PTGS2, ZFP36) were also induced by SARS-CoV-1 and SARS CoV2 infection (Figure 8 and Suppl. Figure 1). The induction of these genes was correlated with the induction of interferon- $\beta$ (IFNB1) by the virus infection (Figure 8 and Suppl. Figure 1). TNFSF10/TRAIL is a widely expressed member of the TNF family involved in critical biological functions including apoptosis and tumor suppressor activities and demonstrated to be part of the effector mechanisms by which influenza-specific $\mathrm{T}$ cells protect the host against virus infection [54, 59]. In contrast, SARS-CoV-2 infection of A549 failed to induce Egr-1 expression (data not shown). Recent studies have shown that Angiotensin-converting enzyme 2 (ACE2) functions as ectopeptidase and virus entry receptor and facilitates entry of SARS-CoV1 and SARS-CoV-2 in lung epithelial cells [60]. Expression of ACE2 in A549 cells rescued SARS-CoV-2- mediated induction of IFNB1 and IFN-regulated transcription factors STAT1, STAT2, IRF9, and EGR1 (Figure 9). Furthermore, expression of interferon-stimulated and EGR1-dependent genes such as chemokines (CXCL10, CXCL2, CCL3, and CCL4) cell growth (ATF3 and TNFSF10) were rescued after SARS-CoV-2 infection of A549 cells expressing ACE2 (Figure 9). Importantly, the expression of ACE2 alone in A549 cells did not induce Egr-1 mRNA levels. These results are consistent with the interpretation that virus infection activated production and secretion of IFNB1 in lung epithelial cells resulting in autocrine or paracrine interferon signaling and activation of interferon stimulated genes. Differential regulation of EGR1 by SARS-CoV-2 in Calu-3 and A549 suggest that these cell lines may differ in the expression levels of host factor (ACE2) required for SARS-CoV-2 entry. Pandemic 1918 H1N1 influenza virus infection induced expression of Egr-1 and Egr-1 dependent chemokines in mouse lungs [51]. In contrast, influenza A virus (IAV) suppressed Egr-1 expression in NHBE bronchial epithelial cells that is dependent on viral non-structural protein or NS1 (Supplementary Figure 3A). These studies suggest that multiple factors such as influenza strain, virus- encoded factors (NS1), and cell type regulate Egr-1 expression. Respiratory syncytial virus (RSV) or parainfluenza virus (HPIV3) infection also induced Egr-1 mRNA in A549 cells, 24 hours post-infection (Supplementary Figure 3B). These studies suggest that Egr-1 expression is a common host response to many respiratory viruses. A snapshot of Egr-1 regulation by multiple respiratory viruses was shown (Figure 10).

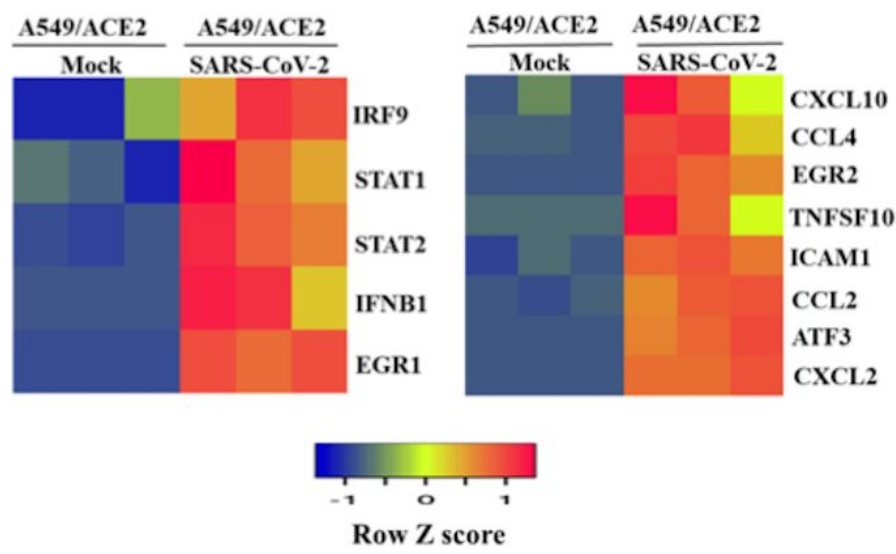

Figure 9: Regulation of type I interferon-stimulated transcription factors and interferon-stimulated genes (ISG) by SARS-CoV-2 in A549 cells expressing ACE2 receptors. A549 cells expressing ACE2 receptors were mock- infected or infected with SARSCoV-2 virus for 24 hours. RNA expression levels were normalized by DEseq2. Data from three samples for each condition were shown. 


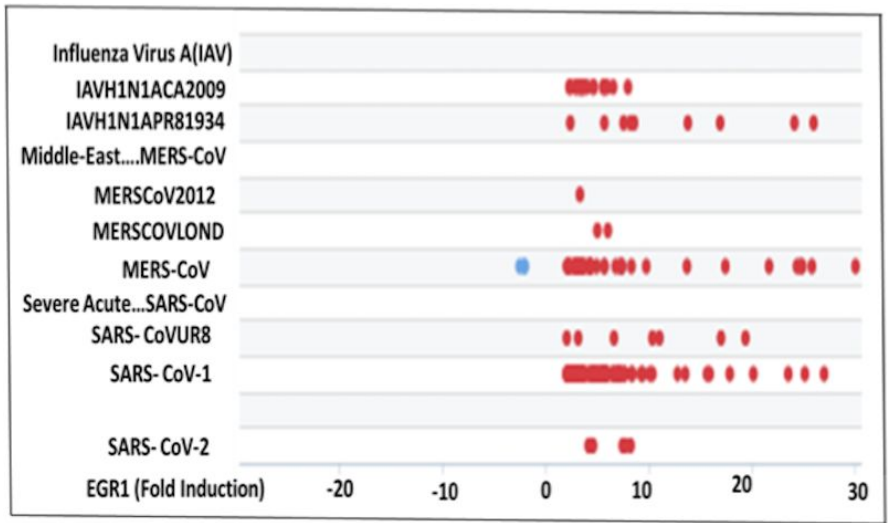

Figure 10: A snapshot of the regulation of Egr-1 RNA levels by respiratory viruses represented in the microarray datasets. Induction or suppression of Egr-1 RNA levels were shown by red and blue ovals, respectively. Each oval represents a separate experiment.

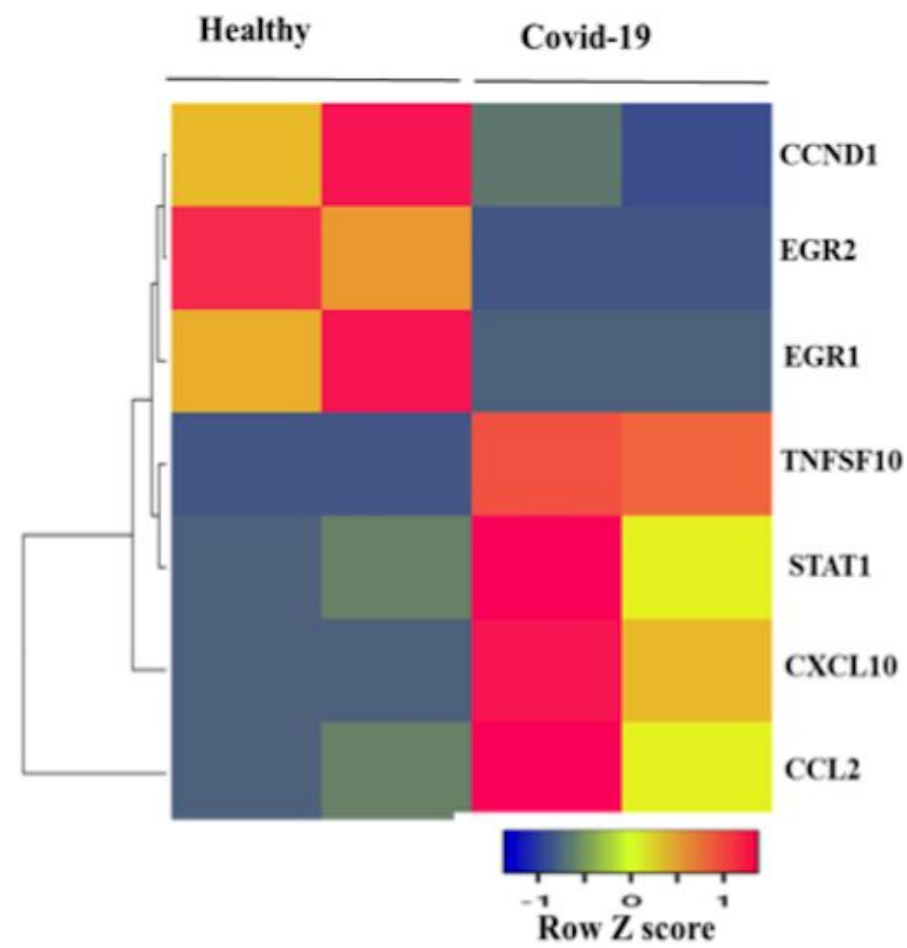

Figure 11: Relative mRNA expression levels of Egr-1 and interferon-stimulated genes in the lung biopsies of healthy controls and COVID-19 patients. Healthy and COVID-19 samples were represented by blue and red, respectively. RNA expression values were normalized by DEseq2. Data from two samples for each condition were shown.

\subsection{Regulation of Egr-1 in COVID-19 patient lung biopsies}

Gene expression profiling studies of a limited number of human lung biopsies and PBMC of healthy controls and COVID-19 patients were reported recently [30, 31]. Interrogation of the gene expression data in lung biopsies revealed that Egr-1 expression levels were significantly lower in COVID-19 patients compared with healthy 
A

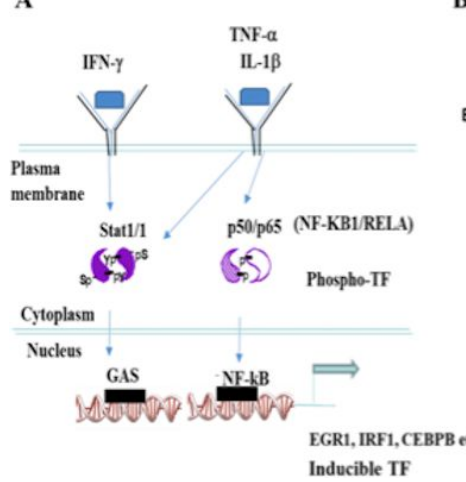

B

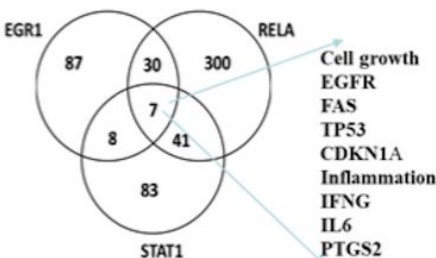

Figure 12: Identification of common target genes of EGR1, STAT1 and RELA (A) Schematic outline of IFN- $\gamma$, TNF- $\alpha$, and IL-1 $\beta$ signaling (B) Common gene targets of Egr-1, STAT1, and RELA were represented by a Venn diagram.

controls. Expression of Egr-1 target genes related to cell growth such as CCND1 and EGR2 were also downregulated. In contrast, expression of Egr-1 target genes involved in inflammatory responses such as CXCL10, CCL2, and TNFSF10 were dramatically enhanced in COVID-19 patients compared with healthy controls (Figure 11). Interestingly, these inflammatory genes were also regulated by STAT1. Consistent with this interpretation, STAT1 expression levels were significantly increased in COVID-19 patients compared with healthy controls ((Figure 11). There are several limiting factors to interpreting the data. The lung is a complex tissue consisting of more than thirty cell types with differential contributions with respect to cell mass and gene expression. For example, type I and type II cells in mouse lungs constitute the major and minor cell types and express distinct cell markers [35]. Without information on the expression levels in distinct cell types, it is difficult to correlate Egr-1 expression with target genes in the whole lung tissue. In support of this view, in a mouse model of $\mathrm{CD}^{+} \mathrm{T}$ cell -mediated lung injury, chemokine expression was dependent on Egr-1 activation in alveolar type II cells [63]. Another intriguing possibility is that enhanced STAT1 expression compensates for decreased Egr1 expression in some cell types in the lung. It is important to consider that in addition to changes in mRNA levels, the phosphorylation status of STAT1 and Egr-1 may play an important role in the transcriptional regulation of chemokine target genes. It is likely that differential expression in distinct cell types may account for the disparity of Egr-1 and its target gene expression in healthy controls and COVID-19 patients. Consistent with our results, recent multiplex antibody array and gene expression profiling and studies in COVID-19 patients have confirmed that inflammatory cytokines (IFN- $\gamma$, TNF- $\alpha$, and IL- $\beta$ ), chemokines (CXCL10, CCL2, CCL3, CCL4) and apoptosis (TNFSF10TRAIL) were dramatically elevated in the lungs and correlated with the severity of the disease $[57,58]$. Imbalanced antiviral and proinflammatory cytokine responses were implicated in the pathogenesis of COVID-19 [30]. TNF- $\alpha$ and IL- $\beta$ are involved in the activation of nuclear factor-kB composed of NFKB1 (p50) and RELA (p65) while IFN- $\gamma$ activates STAT1 homodimers by phosphorylation within 30 minutes and induce transcription factors such as EGR1, IRF1 and CEBPB that sustain secondary responses (Figure 12A). Transcription factors activated in multiple signaling pathways involved in a common biologic function often share common protein interaction partners and target genes as revealed by pair wise analysis of critical hubs [47]. Bioinformatic analysis of common protein interaction partners by pair wise analysis revealed that STAT1, RELA and EGR1 share BRCA1 and NTRK1 in the network (Suppl. data Fig 3). Furthermore, these transcription factors also share common target genes involved in cell growth such as TP53, FAS, and CDKN1A and inflammation such as IL-6 and PTGS2 (Figure 12B). Inhibiting common protein interaction partners or common target genes involved in inflammatory responses may have therapeutic potential in the treatment of COVID-19. 


\section{Conclusion}

Egr-1 has emerged as a key regulator of cell growth, reproduction, and response to tissue injury [16, 61, 62, 63]. Egr-1 was rapidly induced by interferons and pro-inflammatory cytokines such as TNF- $\alpha$, and IL-1 $\beta[15,63,64]$. Recent studies have demonstrated dramatic changes in type I interferon, TNF- $\alpha$, and IL- $1 \beta$ production by immune cells and cytokine-mediated lung inflammation in COVID-19 patients [30, 57, 65, 66]. However, the role of Egr-1 in innate immunity and antiviral response to respiratory viruses in general and SARS-CoV-2, in particular, remains to be investigated. Transcriptional factor profiling in the transcriptome revealed that Egr-1was induced by IFN- $\alpha / \beta$, TLR ligands, and TNF- $\alpha$ in human and mouse cells. Studies in mutant cell lines lacking Stat1, Stat2, and Irf9 revealed that IFN- $\alpha / \beta$ induction of Egr-1 was independent of ISGF-3 components and mediated by the activation of Erk-1/2 pathway. Furthermore, respiratory viruses such as SARS-CoV-2 induced production and secretion of IFN- $\beta$ in lung epithelial cells. Autocrine or paracrine interferon signaling induced Egr-1 and its target genes in several lung epithelial cell lines and in COVID-19 patients. Activation of Egr-1 by IFN- $\alpha / \beta$ and TNF- $\alpha$ and cross-talk between the pathways modulates signal transduction and inflammatory response in innate immunity.

Acknowledgements: We would like to thank Dr. George Stark (Lerner Research Institute, The Cleveland Clinic Foundation) and N.S. Sastry (Chelmsford, MA, USA) for continuous support. We thank Chelmsford Library for computer facilities.

Financial Support: This research received no specific grant from any funding agency, commercial or nonprofit sectors.

Conflict of Interests Statement: The authors have no conflicts of interest to disclose.

Ethics Statement: This research did not required ethical approval.

\section{References}

[1] Stark, G. R., Kerr, I. M., Williams, B. R., et al. (1998). How cells respond to interferons. Annu Rev Biochem, 67, 227-264.

[2] Stark, G. R., Darnell, J. E. Jr. (2012). The JAK-STAT pathway at twenty. Immunity, 36, 503-514.

[3] Stark, G. R., Friedman, R. L., McMahon, M., et al. (1984). Induction of human mRNAs by interferon. Philos Trans R Soc Lond B Biol Sci, 307, 227-230.

[4] Ramana, C. V., Chatterjee-Kishore, M., Nguyen, H., Stark, G. R. (2000). Complex roles of Stat1 in regulating gene expression. Oncogene, 19, 2619-2627.

[5] Majoros, A., Platanitis, E., Kernbauer-Hölzl, E., Rosebrock, F., Müller, M., Decker, T. (2017). Canonical and Non-Canonical Aspects of JAK-STAT Signaling: Lessons from Interferons for Cytokine Responses. Front Immunol,; 8, 29.

[6] Platanias, L (2005). Mechanisms of type- I- and Type-II-interferon-mediated signaling. Nature Rev Immunol, 5, 375-386.

[7] Ramana, C. V., Gil, M. P., Schreiber, R. D., Stark, G. R. (2002). Stat1-dependent and-independent pathways in IFN gamma -dependent signaling. Trends Immunol, 23, 96-101.

[8] Kumar, H., Kawai, T., Akira, S. (2009). Toll-like receptors and innate immunity. Biochem Biophys Res Commun, 388, 621-625.

[9] Adelaja, A., Hoffmann, A. (2019). Signaling Crosstalk Mechanisms That May Fine-Tune Pathogen-Responsive NFkB. Front Immunol, 10, 433

[10] Bachmann, A., Hanke, B., Zawatzky, R., et al. (2002). Disturbance of tumor necrosis factor alpha-mediated beta interferon signaling in cervical carcinoma cells. J Virol, 76, 280-291.

[11] Ramana, C. V., DeBerge, M.P., Kumar, A., Alia. C.S., Durbin, J. E., Enelow, R. I. (2015). Inflammatory impact of IFN- $\gamma$ in CD8+ T cell-mediated lung injury is mediated by both Stat1-dependent and - independent pathways. Am J Physiol Lung Cell Mol Physiol, 308, L650-L657.

[12] Palucka, A. K., Blanck, J P.. Bennett, L.. Pascual, V., Banchereau, J. (2005). Cross-regulation of TNF and IFN- $\alpha$ in autoimmune disease. Proc Natl Acad Sci USA, 102, 3372-3377.

[13] Banchereau, J., Pascual, V. (2006). Type I Interferon in systemic lupus erythematosus and other autoimmune diseases. Immunity, 25, 383-392. 
[14] Gashler, A. L., Sukhatme, V. P. (1995). Early growth response protein 1(Egr-1): prototype of a zinc-finger family of transcription factors. Prog Nucleic Acids Res Mol Biol., 150, 191-224.

[15] Cao, X., Guy, G. R., Sukhatme, V. P., Tan, Y. H. (1992). Regulation of the Egr-1 gene by tumor necrosis factor and interferons in primary human fibroblasts. J Biol Chem, 267, 1345-1349.

[16] Thiel, G., Cibelli, G. (2002).Regulation of life and death by the zinc finger transcription factor Egr-1. J Cell Physiol, 193, 287292.

[17] Silverman, E.S., De Sanctis, G.T., Boyce, J., et al. (2001). The transcription factor early growth-response factor 1 modulates tumor necrosis factor-alpha, immunoglobulin E, and airway responsiveness in mice. Am J Respir Crit Care Med, 163, 778-785.

[18] Zhang, W., Yan, S. D., Zhu, A., et al. (2000). Expression of Egr-1 in late stage emphysema. Am J Pathol, 157, $1311-1320$.

[19] Lee, C. G., Cho, S. J., Kang, M. J., et al. (2004). Early growth response gene 1-mediated apoptosis is essential for transforming growth factor beta1-induced pulmonary fibrosis. J Exp Med, 200, 377-389.

[20] Yan, S. F., Fujita, T., Lu, J., et al. (2001). Egr-1, a master switch coordinating upregulation of divergent gene families underlying ischemic stress. Nat. Med, 12, 1355-1361.

[21] Cho, S. J., Kang, M.J., Homer, R. J., et al. (2006). Role of early growth response -1(Egr-1) in interleukin-13-induced inflammation and remodeling. J Biol Chem, 281, 8161-8168.

[22] McCaffrey, T.A., Fu, C., Du, B., et al. (2000). High level expression of Egr-1 and Egr-1 inducible genes in mouse and human atherosclerosis. J Clin Invest 105, 653-662.

[23] Pritchard, M.T., Nagy, L.E. (2005). Ethanol-induced liver injury: potential roles for EGR-1. Alcoholism: Clin and Exp Res, (suppl). 29, 146S-150S.

[24] Ji, B., Chen, X., et al. (2003). Pancreatic gene expression during the initiation of acute pancreatitis: identification of EGR-1 as a key regulator. Physiol Genomics, 14, 59-72.

[25] Kim, N. D., Moon, J-OK., et al. (2006). Early growth response factor-1 is critical for cholestatic liver injury. Toxicol Sci, 90, 586-595.

[26] Guha, M., O'Connell, M.A., Pawlinski, R., et al. (2000). Lipopolysaccharide activation of the Mek-ERK1/2 pathway in human monocytic cells mediates tissue factor and tumor necrosis factor a expression by inducing Elk-1 phosphorylation and Egr-1 expression. Blood, 98, 1429-1439.

[27] Waddell, S. J., Popper, S. J., Rubins, K. H, et al. (2010). Dissecting interferon-induced transcriptional programs in human peripheral blood cells. PLoS One, 5, e9753.

[28] Ramirez-Carrozzi, V. R., Braas, D., Bhatt, D. M., et al. (2009). A unifying model for the selective regulation of inducible transcription by $\mathrm{CpG}$ islands and nucleosome remodeling. Cell, 138, 114-128.

[29] Bolen, C R., Ding, S., Robek, M.D., Kleinstein, S.H. (2014). Dynamic expression profiling of type I and type III interferonstimulated hepatocytes reveals a stable hierarchy of gene expression. Hepatology, 59, 1262-1272.

[30] Blanco-Melo, D., Nilsson-Payant, B.E., Liu, W. C., Uhl, S., Hoagland D., (2020). Imbalanced Host Response to SARS-CoV-2 Drives Development of COVID-19. Cell, 181, 1036-1045.e9.

[31] Wilk A. J., Rustagi, A., Zhao N. Q., Roque, J., (2020). A single-cell atlas of the peripheral immune response in patients with severe COVID-19. Nat Med, 7, 1070-1076.

[32] Kota, R .S., Rutledge, J. C., Gohil, K., Kumar, A., Enelow, R. I., Ramana, C. V. (2006). Regulation of gene expression in RAW 264.7 macrophage cell line by interferon-gamma. Biochem Biophys Res Commun, 342(4), 1137-1146.

[33] Rani, M. R., Foster, G. R., Leung, S., et al. (1996). Characterization of beta-R1, a gene that is selectively induced by interferon beta (IFN-beta) compared with IFN-alpha. J Biol Chem, 271, 22878-22884.

[34] Ramana, C.V., Gil, M. P., Han, Y., Ransohoff, R. M, Schreiber, R. D., Stark, G. R. (2001). Stat1-independent regulation of gene expression in response to IFN-gamma. Proc Natl Acad Sci U S A, 98(12), 6674-6679.

[35] Ramana, C. V. (2019). Insights into the signal transduction pathways of mouse lung type II cells revealed by Transcription Factor Profiling in the transcriptome. Genomics Inform, 17(1):e8.

[36] Kumar, H., Kawai, T., Akira, S. (2011). Pathogen recognition by the innate immune system. Int Rev Immunol, 2011, 30(1), 16-34.

[37] Morrison, D. K. MAP kinase pathways. (2012). Cold Spring Harb Perspect Biol, 4(11):a011254.

[38] Chai, J., Tarnawski, A.S. (2002). Serum response factor: discovery, biochemistry, biological roles and implications for tissue injury healing. J Physiol Pharmacol, 53(2), 147-157.

[39] Stancato, L. F., Sakatsume, M., David, M., et al. (1997). Beta interferon and oncostatin M activate Raf-1 and mitogen-activated protein kinase through a JAK1-dependent pathway. Mol Cell Biol, 17(7), 3833-3840.

[40] Joshi, S., Kaur, S., Redig, A. J., et al. (2009). Type I interferon (IFN)-dependent activation of Mnk1 and its role in the generation of growth inhibitory responses. Proc Natl Acad Sci U S A, 106(29), 12097-12102.

[41] Livingstone, M., Sikström, K., Robert, P. A., et al. (2015). Assessment of mTOR-Dependent Translational Regulation of Interferon Stimulated Genes. PLoS One, 10, e0133482.

[42] Xie, L., Sullivan, A. L., Collier, J. G., Glass, C. K. (2013). Serum response factor indirectly regulates type I interferon-signaling in macrophages. J Interferon Cytokine Res, 33, 588-596.

[43] Duan, W. R., Ito, M., Park, Y., et al. (2002). GnRH regulates early growth response protein 1 transcription through multiple promoter elements. Mol Endocrinol. 2002, 16, 221-233. 
[44] Iwasaki, A., Medzhitov, R. (2015). Control of adaptive immunity by the innate immune system. Nat Immunol, 2015, 16, 343353.

[45] Julkunen, I., Sareneva, T., Pirhonen, T., et al. (2001). Molecular pathogenesis of influenza A virus infection and virus-induced regulation of gene expression. Cytokine growth factor Rev, 12: 171-180.

[46] Han, H., Cho, J.W., Lee, S., et al. (2018). TRRUST v2: an expanded reference database of human and mouse transcriptional regulatory interactions. Nucleic Acids Res, 46, D380-D386.

[47] Ramana, C. V. (2019). Insights into functional connectivity in mammalian signal transduction pathways by pairwise comparison of protein interaction partners of critical signaling hubs. bioRxiv DOI: ?10.1101/2019. 12.30.891200.?

[48] Zhou, P., Yang, X. L, Wang, X.G., et al. (2020). A pneumonia outbreak associated with a new coronavirus of probable bat origin. Nature, 579, 270-273.

[49] Michael, P., Brabant, D., Bleiblo, F., et al. (2013). Influenza A induced cellular signal transduction pathways. J Thorac Dis, 5 (Suppl 2), S132-S141.

[50] Srikiatkhachorn, A., Chintapalli, J., Liu, J., et al. (2010). Interference with intraepithelial TNF- $\alpha$ signaling inhibits CD8 (+) T-cell-mediated lung injury in influenza infection. Viral Immunol, 23, 639-645.

[51] Kash, J. C., Tumpey, T. M., Proll, S. C., et al. (2006). Genomic analysis of increased host immune response and cell death responses induced by 1918 influenza virus. Nature, 443: 578-581.

[52] Walters, K A., D’Agnillo, F., Sheng, Z. M., et al. (2016). 1918 pandemic influenza virus and Streptococcus pneumoniae coinfection results in activation of coagulation and widespread pulmonary thrombosis in mice and humans. J Pathol, 238, 85-97.

[53] Ludwig, S., Ehrhardt, C., Neumeier, E. R., et al. (2001). Influenza virus- induced AP-1-dependent gene expression requires activation of the JNK signaling pathway. J Biol Chem, 276, 10990-10998.

[54] Wurzer, W. J., Ehrhardt, C., Pleschka, S., et al. (2004). NF-kappaB-dependent induction of tumor necrosis factor-related apoptosis -inducing ligand (TRAIL) and Fas/fasL is crucial for efficient influenza propagation. J Biol Chem, 279, 30931-30937.

[55] Pleschka, S., Wolff, T., Ehrhardt, C., et al. (2001). Influenza virus propagation is impaired by inhibition of the Raf/Mek/ERK signaling cascade. Nature Cell Biol, 3, 301-305.

[56] Olschläger, V., Pleschka, S., Fischer, T., et al. (2004). Lung-specific expression of active Raf kinase results in increased mortality of influenza A virus-infected mice. Oncogene, 23, 6639-6646.

[57] Lucas, C., Wong, P., Klein, J., Castro, T. B. R., Silva, J., et. al. (2020). Longitudinal analyses reveal immunological misfiring in severe COVID-19. Nature, 584, 463-469.

[58] Arunachalam, P. S., Wimmers, F., Mok, C. K. P., et al. (2020) Systems biological assessment of immunity to mild versus severe COVID-19 infection in humans Science, 369, 1210-1220.

[59] Hamada, H., Bassity, E., Flies, A., Strutt, T. et al. (2013). Multiple redundant effector mechanisms of CD8+ T cells protect against influenza infection. J Immunol, 190, 296-306.

[60] Li, W., Moore, M. J, Vasilieva, N., et al. (2003). Angiotensin - converting enzyme 2 is a functional receptor for the SARS coronavirus. Nature, 426, 450-454.

[61] Ramadas, N., Rajaraman, B., Kuppuswamy, A. A., Vedantham, S. (2014). Early growth response-1 (EGR-1) - a key player in myocardial cell injury. Cardiovasc Hematol Agents Med Chem, 12(2), 66-71.

[62] Yan, S. F., Harza, E., et al. (2006). Protein kinase C beta/early growth response-1 pathway. A key player in ischemia, atherosclerosis, and restenosis. J Am Coll Cardiol, 48, (suppl), A47-A55.

[63] Ramana, C. V., Cheng, G. S., Kumar, A., Kwon, H. J., Enelow, R. I. (2009). Role of alveolar epithelial early growth response 1(Egr-1) in CD8+ T cell-mediated lung injury. Mol Immunol, 47, 623-631.

[64] Hoffmann, E., Ashouri, J., Wolter, S., et al. (2008). Transcriptional regulation of EGR-1 by the interleukin-1-JNK-MKK7-c-Jun pathway. J Biol Chem, 283, 12120-12128.

[65] Hadjadj, J., Yatim, N., Barnabei, L., et al. (2020). Impaired type I interferon activity and inflammatory responses in severe COVID-19 patients. Science, eabc6027. doi:10.1126/science.abc6027.

[66] Lee JS, Park S, Jeong HW, et al. (2020). Immunophenotyping of COVID-19 and influenza highlights the role of type I interferons in development of severe COVID-19. Sci Immunol, 5(49):eabd1554. doi:10.1126/sciimmunol.abd1554

\section{Supplementary data}

Suppl. Fig 1. Regulation of interferon-stimulated gene (ISG) expression by Coronaviruses in Calu-3 cells. Calu-3 cells were mock- infected or infected with the virus (SARS-CoV-1 or SARS-CoV-2) for 4-24 hours. Average fold induction from two samples for each condition were shown. RNA expression levels were normalized by DEseq2. 
Suppl. Fig 2. Time-course analysis of Egr-1 RNA levels in human airway epithelial (HAE1) cells by SARS-CoV-1. Egr-1 expression levels of in HAE1 cells in response to SARS-CoV-1 infection, after 0-96 hours post infection (hpi) were shown. Data from one sample for each condition were shown. Expression levels were normalized to mock-infection.

Suppl. Fig 3. Regulation of Egr-1 mRNA levels by influenza viruses, respiratory syncytial virus (RSV) and parainfluenza virus (HPIV3). (A) Human NHBE1 bronchial epithelial cells were mock- infected or infected with influenza virus (IAV) or influenza virus lacking NS1 (IAVNS1) for 12 hours. (C) Human A549 lung cells were mock- infected or infected with the RSV or PIV3 for $24 \mathrm{hrs}$. Egr-1 mRNA levels were normalized by DESeq2 and expressed as fold induction from triplicate samples.

Suppl. Fig. 4. Identification of common protein interaction partners of EGR1, STAT1 and RELA by pairwise analysis (A) Common protein interaction partners of EGR1, STAT1, and RELA were represented by a Venn diagram (B) Network representation of common interaction partners of EGR1, STAT1, and RELA visualized using BIOGRID software tools. Protein interactions and genetic interactions in the network were represented by orange and green, respectively. 